Paper for the Special Session on Nanostructured Materials at the $49^{\text {th }}$

AIAA/ASME/ASCE/AHS/ASC

Structures, Structural Dynamics and Materials Conference and Exhibit

\title{
New Developments in the Embedded Statistical Coupling Method: Atomistic/Continuum Crack Propagation
}

\author{
E. Saether ${ }^{*}$, V. Yamakov ${ }^{\dagger}$, and E. Glaessgen ${ }^{\text {* }}$ \\ NASA Langley Research Center, Hampton, VA, 23681
}

\begin{abstract}
A concurrent multiscale modeling methodology that embeds a molecular dynamics (MD) region within a finite element (FEM) domain has been enhanced. The concurrent MD-FEM coupling methodology uses statistical averaging of the deformation of the atomistic MD domain to provide interface displacement boundary conditions to the surrounding continuum FEM region, which, in turn, generates interface reaction forces that are applied as piecewise constant traction boundary conditions to the MD domain. The enhancement is based on the addition of molecular dynamicsbased cohesive zone model (CZM) elements near the MD-FEM interface. The CZM elements are a continuum interpretation of the tractiondisplacement relationships taken from MD simulations using Cohesive Zone Volume Elements (CZVE). The addition of CZM elements to the concurrent MD-FEM analysis provides a consistent set of atomistically-based cohesive properties within the finite element region near the growing crack. Another set of CZVEs are then used to extract revised CZM relationships from the enhanced ESCM simulation of an edge crack under uniaxial loading.
\end{abstract}

\footnotetext{
* Durability, Damage Tolerance, and Reliability Branch, MS/188E, AIAA Member.

${ }^{\dagger}$ National Institute for Aerospace.

‡ Durability, Damage Tolerance, and Reliability Branch, MS/188E, AIAA Associate Fellow.
} 


\section{Introduction}

Physics-based modeling of fracture begins at nanometer length scales in which atomistic simulation is used to predict the formation, propagation, and interaction of fundamental damage mechanisms. These mechanisms include dislocation formation and interaction, interstitial void formation, and atomic diffusion. The development of these damage mechanisms progress into microscale processes such as local plasticity and small crack formation. Ultimately, damage progression leads to macroscopic failure modes such as plastic yielding and large cracks exhibiting mixed-mode deformation.

Modeling atomistic processes quickly becomes computationally intractable as the system size increases. With current computer technology, the computational demands of modeling suitable domain sizes (on the order of hundreds of atoms for quantum mechanics-based methods, and potentially billions of atoms for classical mechanics-based methods) and integrating the governing equations of state over sufficiently long time intervals quickly reaches an upper bound for practical analyses. In contrast, continuum mechanics methods such as the finite element method (FEM) provide an economical numerical representation of material behavior at length scales in which continuum assumptions are appropriate. Thus, multiscale modeling strategies are needed using the most efficient material model at different length scales for the purpose of developing a unified description of the hierarchy of processes that govern fracture.

The concept of bridging length scales by coupling different computational paradigms is particularly attractive as a highly efficient means of reducing the computational cost of the simulations in cases that require the modeling of relatively large material domains to capture the complete deformation field, but where atomic and subatomic refinement is needed only in very localized regions (e.g., near a crack tip or dislocation core). Such computational issues arise in modeling crack nucleation and propagation, and in modeling dislocation formation and interaction. Coupled models allow the size limitations of the atomistic simulation to be avoided by embedding an inner domain, where complex dynamic processes and large deformation 
gradients exist, within an outer domain, where the deformation gradients are small so that a continuum representation of the material becomes appropriate.

Over the past decade, various methods that directly couple material representations at different levels of refinement have been developed and offer significant computational advantages compared to full atomistic simulations for predicting deformation and fracture processes. ${ }^{1-11}$ A common feature of all these approaches for coupling atomistic regions with continuum domains is the refinement of the FEM mesh down to the atomic scale However, a robust connection between continuum and discrete quantities can be achieved through a statistical averaging over scales where the discreteness of the atomic structure can be successfully approximated as a continuum. With the use of statistical averages to couple the two computational schemes, the developed approach constitutes a statistical coupling approach, and the developed MD-FEM coupling method is referred to as the embedded statistical coupling method (ESCM). ${ }^{12,13}$ The resulting model that consists of an MD system embedded in a FEM domain is depicted in Figure 1.

The statistical averaging over both time and volume of atomistic subdomains at the MD-FEM interface provides nodal displacement boundary conditions to the continuum FEM domain, which, in turn, generates interface reaction forces that are applied as constant traction boundary conditions $^{14}$ to the atoms within the localized MD subdomain. This process is iterated until the averaged quantities reach a stationary value. Typically, one finite element at the interface encompasses a region of several hundred to several thousand atoms. At this scale, the discreteness of the atomic structure is homogenized enough so that the FEM domain responds to the atomistic domain as an extension of the continuum. However, a moving crack that grows near the transition between the atomistic domain and the finite element region experiences an artificial constraint because using standard continuum finite elements along the crack path can not simulate the fracture process within the continuum.

One means of accommodating the deformation within the finite element domain near the MDFE interface is through the incorporation of cohesive zone model (CZM) elements in the analysis. Cohesive zone models approximate debonding processes along an interface ${ }^{15}$ and are 
frequently used in conjunction with finite element approaches to study fracture in a wide variety of materials. Recently, Cohesive Zone Volume Elements (CZVEs) ${ }^{16}$ have been introduced in the MD analysis to calculate atomistic-based parameters defining CZMs. The resulting CZMs exhibit the aggregate behavior of a volume of atoms in the MD simulation. ${ }^{16-18}$ By placing the CZM elements along the plane of crack growth within the finite element region of the ESCM model, the fracture process can be simulated in the continuum as the crack approaches the MDFE interface. Figure 2 depicts the coupled MD-FEM model containing an edge crack. CZM elements are identified that represent the cohesive properties of the GB in the continuum. Crystal I and II identify the MD regions that have been rotated through a tilt angle to form the GB.

The present analysis considers intergranular fracture wherein MD simulations of fracture along grain boundaries (GBs) are used to obtain the atomistic response of crack propagation under applied loads along a characteristic length of the GB. Figure 3 shows a typical $<110>\Sigma 99$ symmetric tilt GB formed between two grains in a fcc metal. Common neighbor analysis $(\mathrm{CNA})^{19,20}$ is used to identify atoms in different crystallographic states: fcc (small dots), hcp (triangles), non-crystalline atoms (large dots), and surface atoms (squares), indicating existing vacancies in the GB. The length scale is in units of the lattice constant of Al, $a_{o}=0.405 \mathrm{~nm}$ using the potential of Mishin, et al. ${ }^{21}$

In this paper, an outline of the ESCM approach to coupling MD and FEM computational domains is presented in Section II. Section III summarizes the determination of CZM relationships from MD analysis using CZVEs to simulate fracture at higher length scales. Section IV presents the simulation of an edge crack propagating through a continuum into an atomistic domain where deformation along the crack plane in the continuum region is modeled using CZMs. This simulation provides atomistic resolution of near-tip processes in a crack with an extent that could not be simulated using purely MD methods. Finally, Section V presents a summary of the analysis and overall methodology. 


\section{The ESCM Model}

The structure of the basic ESCM model consists of four regions that are depicted in Figure 4. In this model, an Inner MD Region is connected to a FEM Domain along an interfacing segment in which the two material representations overlap. This segment is divided into an Interface MD Region that, together with the superposed FE nodes, constitutes the MD/FE Interface, and a Surface MD Region that does not interact with superposed FEM nodes but is used to compensate atomic free edge effects. The following subsections outline the function of each of these regions and the algorithmic coupling used in the ESCM. Complete details of the basic method are presented by Saether et al. ${ }^{12,13}$

\section{II.1. MD and FEM model components}

The Inner MD Region is used to model material phenomena at the atomistic level. This Inner MD Region should be large enough to ensure a statistically smooth transition from an atomistic to continuum representation at the Interface MD Region while modeling any of the types of processes (e.g., mostly plastic deformation events such as dislocation or void nucleation, crack propagation) that evolve during the simulation. Together, the Inner, Interface and Surface MD Regions constitute the complete MD system.

The only difference between the three MD regions is that, while the atoms in the Inner MD Region are subject only to the interatomic forces of neighboring atoms, the Interface and Surface MD Regions are also subjected to external forces involved in the ESCM coupling procedure.

The fourth component is a FEM Domain that permits a large reduction in the computational cost of the performed simulations by replacing the atomistic representation with a continuum model in those regions of the system where the deformation gradients are small and atomic-level resolution is not necessary. 


\section{II.2. The MD/FE Interface}

The first role of the MD/FE Interface is to provide a computational linkage between the MD region and FEM domain. The atoms that surround a given FEM node at the interface are partitioned to form a cell in the Interface MD Region, called an Interface Volume Cell (IVC), as shown in Figure 4. The IVCs are used to compute averaged MD displacements at the IVC mass centers that are then prescribed as displacement boundary conditions to the associated interface FEM nodes. During the coupled MD-FEM simulation, a spatial average within each $k^{\text {th }}$ IVC is performed to yield the center of mass displacement $\vec{\delta}_{C M, k}^{M D}$, which is further averaged over a certain period of $M \mathrm{MD}$ time steps to yield the statistical displacement vector, $\vec{\delta}_{I, k}^{M D}$

$$
\vec{\delta}_{I, k}^{M D}=\left\langle\vec{\delta}_{C M, k}^{M D}\right\rangle_{t}=\frac{1}{M} \sum_{j=1}^{M}\left(\vec{r}_{C M, k}\left(t_{j}\right)-\vec{r}_{C M, k}(0)\right)
$$

In the above expression, $\vec{r}_{C M, k}\left(t_{j}\right)=\frac{1}{N_{k}} \sum_{i=1}^{N_{k}} \vec{r}_{i}\left(t_{j}\right)$ is the mass center of the $k^{\text {th }}$ IVC containing $N_{k}$ atoms at positions $\vec{r}_{i}$ at time $t_{j}$ of the $j^{\text {th }}$ MD step. The mass center displacement, $\vec{\delta}_{C M, k}^{M D}$, in (1) is calculated relative to the initial zero-displacement position of the $k^{\text {th }} \operatorname{IVC}, \vec{r}_{C M, k}(0)$.

The second role of the IVCs is to distribute reaction forces from the interface FEM nodes as external forces applied to the corresponding atoms within the IVC. The FEM reaction forces are computed as

$$
\left\{R_{I}\left(t_{n}\right)\right\}=\left[K_{I V}\right]\left\{\delta_{V}\left(t_{n}\right)\right\}+\left[K_{I F}\right]\left\{\delta_{F}\left(t_{n}\right)\right\}+\left[K_{I I}\right]\left\{\delta_{I}\left(t_{n}\right)\right\}
$$

where $K_{I V}, K_{I F}, K_{I I}$ are the terms in the partitioned FEM stiffness matrix representing the interior, far-field and interface regions, respectively. $\delta_{V}, \delta_{\mathrm{F}}$ and $\delta_{\mathrm{I}}$ are the corresponding displacement vectors with $\delta_{I}$ obtained from the MD region (Equation 1). 
A third role of the IVCs, in the Interface MD region, together with the SVCs in the Surface MD region, is to incorporate a dissipative damping mechanism for phonons propagating into the interface. $^{13}$

\section{II.3. The Surface MD Region}

In order for the MD domain to deform freely in response to applied reaction forces, it is modeled using free surface boundary conditions. However, the existence of a free surface introduces several undesirable effects in the MD system. First, it creates surface tension forces that must be removed to avoid distorting the MD response. Second, because atoms that should lie within the cutoff radius of the free surface atom's potential are missing, the coordination number of the surface atoms is reduced and they are, thus, less strongly bonded to the surrounding atomic field than those within the interior. Under sufficiently large reaction forces, these atoms may be separated from the surface layer causing a spurious surface degradation within the MD domain. To mitigate these free surface effects and to stabilize the atoms in the Interface MD Region, a Surface MD Region is introduced as shown in Figure 4.

While the Surface MD Region eliminates the free surface effects within the Inner MD Region, it also introduces an undesirable fictitious stiffness which elastically constrains the deformation of the Inner MD Region. The separate effects of surface tension and the fictitious stiffness cannot be computed independently. However, their combined effect may be defined as a

resultant force, $\vec{f}_{s}$, which acts at the boundary between the Surface and Interface MD Region, and is given by the sum of two components expressed as

$$
\vec{f}_{s}=\vec{\xi}+\vec{\tau}
$$

In Equation 2, $\vec{\xi}$ is the elastic reaction of the Surface MD Region under deformation, and $\vec{\tau}$ is the force that results from the surface tension as shown in Figure 4. 
A way to mitigate both the surface tension and the elastic response of the Surface MD Region is to estimate and compensate for the force, $\vec{f}_{s}$. In the ideal case, when $\vec{f}_{s}$ is fully compensated, the Surface MD Region acts as if it possesses zero stiffness and experiences no surface tension, thereby mitigating spurious influences on the Inner MD Region. Subdividing the Surface MD region into a number of SVCs helps to follow the variations of $\vec{f}_{s}$ along the perimeter of the Interface MD Region. For convenience, the partitioning of SVCs can be made to follow the IVC partitioning of the Interface MD Region. The resultant force is then calculated individually for each SVC. To compensate for these effects, a counterforce, $\vec{f}_{c}$, is computed along the IVC/SVC interface and then distributed over the atoms of each SVC in a similar manner as the nodal reaction forces are applied to the IVCs of the Interface MD Region. The calculation of the counterforce, $\vec{f}_{c}$, is presented in Saether, et al. ${ }^{12,13}$

\section{II.4. MD-FEM coupling}

The MD-FEM coupling in the ESCM is achieved through an iterative equilibration scheme between the MD system and the FEM Domain. In this scheme, iterations begin with displacements at the $\mathrm{MD} / \mathrm{FE}$ Interface that are calculated as statistical averages over the atomic positions within each IVC and averaged over the time of the MD simulation. These average displacements are then imposed as displacement boundary conditions, $\left\{\vec{\delta}_{I}\right\}$, on the FEM domain. The resulting FEM boundary value problem is then solved to recover new interface

reaction forces, $\left\{\vec{R}_{I}\right\}$, resulting from the applied interface displacements and any imposed farfield loading. The new interface reaction forces, $\left\{\vec{R}_{I}\right\}$, are then distributed to the atoms in the IVCs, thus defining new constant traction boundary conditions on the MD system. This MDFEM iteration cycle repeats until a stable equilibrium of both displacements and forces between the atomistic and continuum material fields is established at the interface. Details on calculating the interface reaction forces have been presented elsewhere. ${ }^{12,13}$

\section{II.5. Constraint at MD-FEM interface}


The ESCM was shown in References 12 and 13 to have many desirable features, including the applicability to three-dimensional domains, relatively coarse discretization of the FEM domain, and the ability to consider arbitrary temperature states. Additionally, the methodology was verified using a series of increasingly complex models that included the simulation of a propagating crack within the MD domain. However, a propagating crack that grows near the transition between the atomistic domain and the finite element region experiences an artificial constraint because the finite elements along the crack path cannot properly simulate the fracture process within the continuum, nor can they account for the deformation field (i.e., dislocation emission) produced by the approaching crack. This constraint places an inherent limitation on the original formulation of the ESCM and restricts its applicability to modeling an extent of crack propagation for which damage processes are entirely contained within the MD region. This limitation can be resolved by adding MD-based cohesive zone models (CZM) within the FEM domain along the anticipated plane of crack growth as shown in Figure 2. Section III summarizes the development of the CZM as formulated in Reference 16.

\section{Development of Traction-Displacement Relationships}

Through the definition of CZVEs shown in Figure 5, the results from MD simulations have been recast to obtain averaged continuum traction-displacement relationships that represent the cohesive interactions along a characteristic length of material. Using a purely MD model of a center crack along the $\Sigma 99$ grain boundary presented in Reference 16, the stress and opening profiles are extracted along the crack growing in the system as shown in Figure 6. The figure shows distinct traction, $\sigma_{y y}^{s}(x)$, and displacement, $\lambda$, profiles for the two crack tips of the center crack. The asymmetric behavior results from the orientation of the crystal lattice of the grains forming the grain boundary. A curve fit of $r^{-1 / 2}$ in Figure 6 is shown as a close approximation to the $\sigma_{y y}^{s}(x)$ values extracted from the MD for the brittle tip and are consistent with the predominantly brittle fracture seen in this direction (left to right in Figure 5). In contrast, plastic deformation processes, including twinning and dislocation emission, were shown to dominate deformation at the ductile crack tip growing in the opposite direction (right to left in Figure 5). ${ }^{16}$ 
The $\sigma_{y y}^{s}(x)$ and $\lambda(x)$ profiles in Figure 6 represent the traction and opening displacement at a single instant of time during crack propagation. If the entire simulation of the interface debonding is divided into $N_{t}$ equal intervals of time $t_{q}\left(q \in\left[0, N_{t}\right]\right)$, numerous similar profiles can be taken of many CZVEs placed along the GB. When plotted as a $\sigma_{y y}^{s}$ vs. $\lambda$ curve, each $\left(\sigma_{y y}^{s}\left(x_{p}, t_{q}\right), \lambda\left(x_{p}, t_{q}\right)\right)$ pair represents a point $\sigma_{y y}^{s}\left(\lambda\left(x_{p}, t_{q}\right)\right)$. After sorting these data points in order of ascending $\lambda$, so that $\lambda_{i}<\lambda_{i+1}$, and taking a moving average (or a consecutive mean),

$$
\tau\left(\lambda_{i}\right)=\frac{1}{(2 M+1)} \sum_{j=-M}^{M} \sigma_{y y}^{s}\left(\lambda_{i+j}\right)
$$

in which the results are averaged over $M$ points backward and $M$ points forward from $\lambda_{i}$, a construction of a statistically representative cohesive zone relationship $\tau(\lambda)$ can be made. Complete details of the modeling, fracture processes, and extraction of the CZM response are given in Yamakov, et al. ${ }^{16}$

\section{Crack Propagation through a Continuum-Atomistic Interface}

A continuum-atomistic model for crack propagation along the $\Sigma 99$ GB shown in Figure 2 and analyzed using the enhanced ESCM analysis is presented. The crack grows as an edge crack in the continuum environment subject to mode I loading conditions and penetrates into the atomistic environment (Figure 7). The continuity of the crack propagation process through the MD-FEM interface is achieved by using the CZM constitutive relation derived for interface debonding based on the pure molecular-dynamics simulations ${ }^{16}$ and discussed in Section III. Note that the edge crack in Figure 7 is shown rotated to indicate that the simulation can be used for interfaces of arbitrary orientation.

The dimensions of the coupled MD-FEM model used for this simulation are $d_{\mathrm{FE}}=900 \mathrm{~nm}$ in the $x$ - and $y$ - directions, with a circular MD domain in the $x$ - $y$ plane of diameter, $d_{\mathrm{MD}}=45 \mathrm{~nm}$. The system thickness in the $z$-direction is $h=2.9 \mathrm{~nm}$ with periodic boundary conditions to emulate a bulk atomic state in this direction. The simulation was performed at a temperature $T=$ $300 \mathrm{~K}$ and maintained by a Nose-Hoover thermostat. ${ }^{22}$ 
An edge crack was inserted along the line of symmetry, $c$, in the FEM mesh which ended with four finite elements on the left side before reaching the MD-FEM interface (Figure 2). These four remaining FEs were connected through CZM elements placed along the crack direction (line $c$ in Figure 2), which meet the atomistic GB at the MD-FEM interface. On the right side of the MD domain, the debonding interface is continued through another line of CZM elements, which extends to the opposite outer boundary of the FEM domain.

The system was subject to uniaxial strain, $\varepsilon_{y y}$, applied as displacement boundary conditions at the outer boundary of the FEM domain. The strain was gradually increased from $0.4 \%$ to $2 \%$ in steps of $0.4 \%$ every 20 MD-FEM iterations (20 ps MD simulation time), allowing the MD system to more smoothly follow the stress increase in the surrounding FEM domain. Figure 8 presents the system configuration and $\sigma_{y y}$ stress state at the end of the second iteration when $\varepsilon_{y y}=$ $0.8 \%$.

The process of crack propagation is depicted in Figure 9 through a set of snapshots, magnified to show the physical configuration of the MD domain in the center of the MD-FEM coupled model. The GB interface in the MD domain is visualized as a line of blue and red atoms, indicating atoms in a disordered or hcp surrounding, respectively, reflecting the non-crystalline structure of the GB interface. In Figures 9(a) and 9(b), the crack propagates through the FEM mesh, sequentially opening the CZM elements placed on its path. In Figure 9(c), the crack tip enters the MD domain. The snapshot has captured the instant when the debonding of the Surface and the Interface MD Regions is accomplished in unison with the opening of the last CZM element. The crack tip is about to enter the inner MD region and to continue its propagation in a fully atomistic way. In Figure 9(d), decohesion of the GB in the inner MD region has begun. Shortly afterward, in Figure 9(e), plastic processes start to develop in terms of nucleation of a pair of dislocations, seen as two short blue lines, marking the extended dislocation cores on both sides of the crack tip. Figure 9(f) presents the crack propagated well inside the MD domain. Active dislocation nucleation has already developed and accompanies the crack's propagation. An important result of this process, shown in Figures 9(a-f), is the preservation of the structural 
continuity between the atomistic and the continuum domains at the MD-FE interface through the entire process of crack propagation from the FEM to the MD systems.

The stress continuity along the MD-FE interface is also well preserved during the entire process. Figure 10(a-f) shows the stress state corresponding to the configurational snapshots presented in Figure 9. The largest discrepancy between the stress of the MD system and the FEM mesh is seen in Figures 10(b) and 10(c) where the dynamics of the MD domain did not converge completely before the next stepwise increase of the strain in the static FEM domain was applied. The continuity is improved later in the simulation, in Figures 10(d-e), when the crack propagates further within the MD region.

The problem of coupling the dynamic response of the MD domain to the static response of the FEM system is discussed in detail in Saether, et al. ${ }^{13}$ While the dynamic coupling in the ESCM approach cannot be fully achieved without the use of a dynamic FEM simulation, the iterative scheme of coupling, in which the FEM state is continuously updated in accordance with the reported MD displacements, appears to be sufficient to reproduce the evolution of systems with relatively slow dynamics.

In the original purely MD simulation ${ }^{16}$ that was used to obtain CZM relationships, model size restrictions resulted in a finite geometry distortion. This effect was alleviated using the enhanced ESCM simulation, and the brittle Mode I CZM was recomputed. The procedure for the determination of the CZM parameters in a purely MD simulation is the same as that used in the coupled MD-FEM simulations. The insert in the bottom half of Figure 11 shows the schematic diagram of the discretization of the system volume in the MD simulation and the defining of the CZVEs for extracting the parameters for the CZM elements suitable for use in a continuum simulation. A contrast of the CZVE configurations for analyzing the embedded crack using a purely MD analysis and for the edge crack analyzed using ESCM is seen by comparing Figures 5 and 11. A contrast between the stress and opening profiles for both simulations can be seen by comparing Figures 6 and 11. 
The use of the coupled MD-FEM ESCM approach eliminated the finite geometry distortion seen in the purely MD simulation. ${ }^{16}$ A comparison of the recomputed brittle Mode I CZM with the initial pure MD results from Reference 16 is presented in Figure 12. As shown, the peak stress is increased slightly but the overall energy and functional form of the CZM is closely reproduced.

\section{SUMMARY}

A coupled MD-FEM analysis of intergranular fracture based on the embedded statistical coupling method (ESCM) is presented. The ESCM approach is based on solving the boundary value problem through an iterative procedure for both MD and FEM systems at their common interface. The FEM system is loaded along the MD-FEM interface by nodal displacement boundary conditions obtained as statistical averages of the atomic positions in the MD system at the mass centers of associated Interface Volume Cells (IVCs). The MD system is simulated under constant traction boundary conditions obtained from the FEM system as reaction forces to the MD displacements at the interface. This approach allows the continuity at the MD-FEM interface to be achieved at different length scales inherent to both systems.

An application of ESCM is presented that utilizes Cohesive Zone Volume Elements (CZVEs) and a moving average approach to determine CZM traction-displacement parameters of a 599 GB in aluminum. The model incorporated an edge crack with the tip propagating into the Inner MD Region of ESCM. The continuity of the crack propagation process through the MD-FEM interface was achieved by the derivation of the CZM constitutive relation for interface debonding using CZVEs and moving averages based on an earlier pure molecular-dynamics simulation. CZM parameters for Mode I fracture were determined and compared with those determined from the original pure MD simulation. For a 'brittle' crack tip, the peak stress was increased slightly in the ESCM simulation but the overall energy and functional form of the CZM was closely reproduced.

The presented technique demonstrates the versatility of the ESCM approach and opens new opportunities for performing multiscale atomistic-continuum modeling of fracture processes 
where the fracture region is not limited to the atomistic domain. Additionally, casting atomisticlevel failure processes into continuum constitutive relations is a necessary step towards developing a truly physics-based multiscale methodology to extend material simulation from nanoscopic to microscopic length scales.

\section{Acknowledgments}

V. Yamakov is sponsored through cooperative agreement NCC-1-02043 with the National Institute of Aerospace.

\section{References}

${ }^{1}$ Abraham, F.F., Broughton, J., Bernstein, N., and Kaxiras, E., "Spanning the Continuum to Quantum Length Scales in a Dynamics Simulation of Brittle Fracture,” Europhys. Lett., 44, 1998, pp. 783-787.

${ }^{2}$ Broughton, J., Abraham, F.F., Bernstein, N., and Kaxiras, E., "Concurrent Coupling of Length Scales: Methodology and Application,” Phys. Rev. B, 60, 1999, pp. 2391-2403.

${ }^{3}$ Curtin, W.A., and Miller, R.E., "Atomistic/Continuum Coupling in Computational Materials Science,” Modelling Simul. Mater. Sci. Eng., 11, 2003, pp. R33 - R68.

${ }^{4}$ Park, H.S. and Liu, W.K., "An Introduction and Tutorial on Multiple-scale Analysis in Solids," Comput. Methods in Appl. Mech. Engrg., 193, 2004, pp. 1733-1772.

${ }^{5} \mathrm{Li}, \mathrm{X}$. and E, W., "Multiscale Modeling of the Dynamics of Solids at Finite Temperature”, $J$. Mech. Phys. Solids, 53, 2005, pp. 1650-1685.

${ }^{6}$ Miller, R.R., and Tadmor, E.B., "The Quasicontinuum Method: Overview, Applications and Current Directions,” J. Comp. Aided Mat. Desgn, 9, 2002, pp. 203-239.

${ }^{7}$ Xiao, S.P., and Belytschko, T., "A Bridging domain method for coupling continua with molecular dynamics,” Comput. Methods Appl. Mech. Engrg., 193, 2004, pp. 1645-1669.

${ }^{8}$ Shilkrot, L.E., Miller, R.E., and Curtin, W.A., "Coupled Atomistic and Discrete Dislocation Plasticity,” Phys. Rev. Lett., 89, 2002, p. 025501-1. 
${ }^{9}$ Gumbsch, P. and Beltz, G.E., "On the Continuum Versus Atomistic Description of Dislocation Nucleation and Cleavage in Nickel,” Mod. Sim. Mat. Sci. and Engrg., 3, 1995, pp. 597-613.

${ }^{10}$ Rudd, R.E., and Broughton, J.Q., "Coarse-Grained Molecular Dynamics and the Atomic Limit of Finite Elements”, Phys. Rev. B 58, 2005, pp. R5893-R5896.

${ }^{11}$ Rudd, R.E., and Broughton, J.Q., "Coarse-grained molecular dynamics: Nonlinear finite elements and finite temperature,” Phys. Rev. B 72, 2005, pp. 144104-1-32.

${ }^{12}$ Saether, E., Yamakov, V., and Glaessgen, E.H, "A Statistical Approach for the Concurrent Coupling of Molecular Dynamics and Finite Element Methods," Proceedings of the 48th AIAA/ASME/ASCE/AHS/ASC Structures, Structural Dynamics, and Materials Conference, AIAA-2007-2169-CP, AIAA, Honolulu, HI, April 23-26, 2007.

${ }^{13}$ Saether, E., Yamakov, V., and Glaessgen, E.H., “An Embedded Statistical Method for Coupling Molecular Dynamics and Finite Element Analyses,” 2008, (in review).

${ }^{14}$ Cleri, F., "Representation of Mechanical Loads in Molecular Dynamics Simulations,” Phys. Rev. B, 65, 2001, 014107-1-6.

${ }^{15}$ Tvergaard, V., and Hutchinson, J.W., "The Relation Between Crack Growth Resistance and Fracture Process Parameters in Elastic-Plastic Solids,” J. Mech. and Phys. Solids, 40, 1992, pp. 1377-1397.

${ }^{16}$ Yamakov, V., Saether, E., Phillips, D. R., and Glaessgen, E. H., "Molecular-Dynamics Simulation-Based Cohesive Zone Representation of Intergranular Fracture Processes in Aluminum,” J. Mech. Phys. Solids, 54, 2006, pp. 1899-1928.

${ }^{17}$ Glaessgen, E.H., Saether, E., Phillips, D.R., and Yamakov, V., "Multiscale Modeling of Grain-Boundary Fracture: Cohesive Zone Models Parameterized from Atomistic Simulations,” Proceedings of the 47th AIAA/ASME/ASCE/AHS/ASC Structures, Structural Dynamics, and Materials Conference, AIAA-2006-1674-CP, AIAA, Newport, RI, May 1-4, 2006

${ }^{18}$ Yamakov, V., Saether, E., Phillips, D. R., and Glaessgen, E. H., "Dynamics of Nanoscale Grain-Boundary Decohesion in Aluminum by Molecular-Dynamics Simulation,” J. Mat. Sci., 42, 2007, pp. 1466-1476.

${ }^{19}$ Honeycutt, J. D., and Andersen, H. C., "Molecular Dynamics Study of Melting and Freezing of Small Lennard-Jones Clusters,” J. Phys. Chem., 91, 1987, pp. 4950-4963. 
${ }^{20}$ Clarke, A.S., and Jónsson, H., "Structural Changes Accompanying Densification of Random Hard-Sphere Packings,” Phys. Rev. E, 47, 1993, pp. 3975-3984.

${ }^{21}$ Mishin, Y., and Farkas, D., “Interatomic Potentials for Monoatomic Metals from Experimental Data and Ab Initio Calculations,” Phys. Rev. B, 59, 1999, pp. 3393 - 3407.

${ }^{22}$ Nose S, “A Unified Formulation of the Constant Temperature Molecular Dynamics Method,” J. Chem. Phys., 81, 1984, p. 511. 


\section{FIGURES}

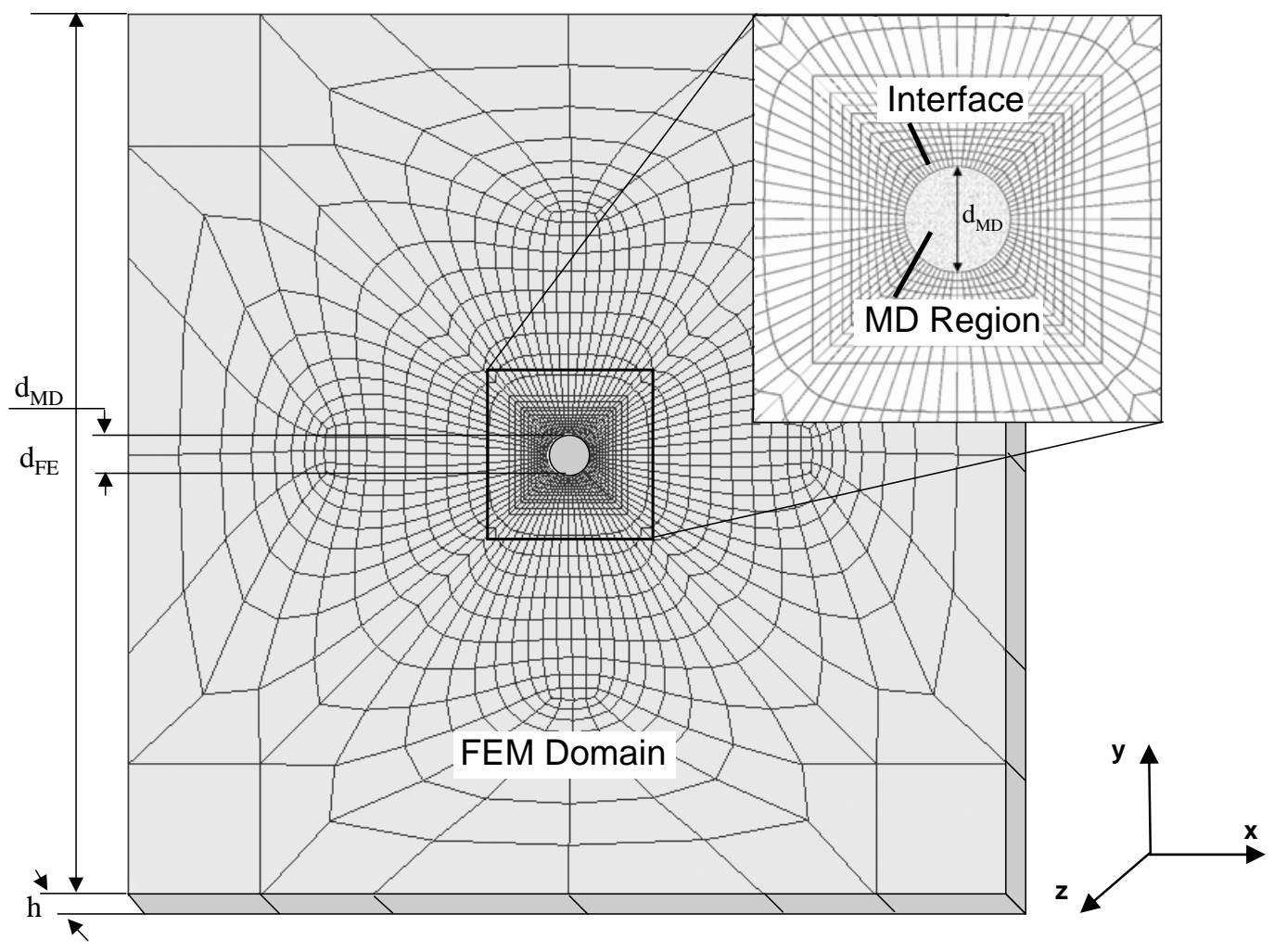

Figure 1. An embedded MD region within a FEM domain. 


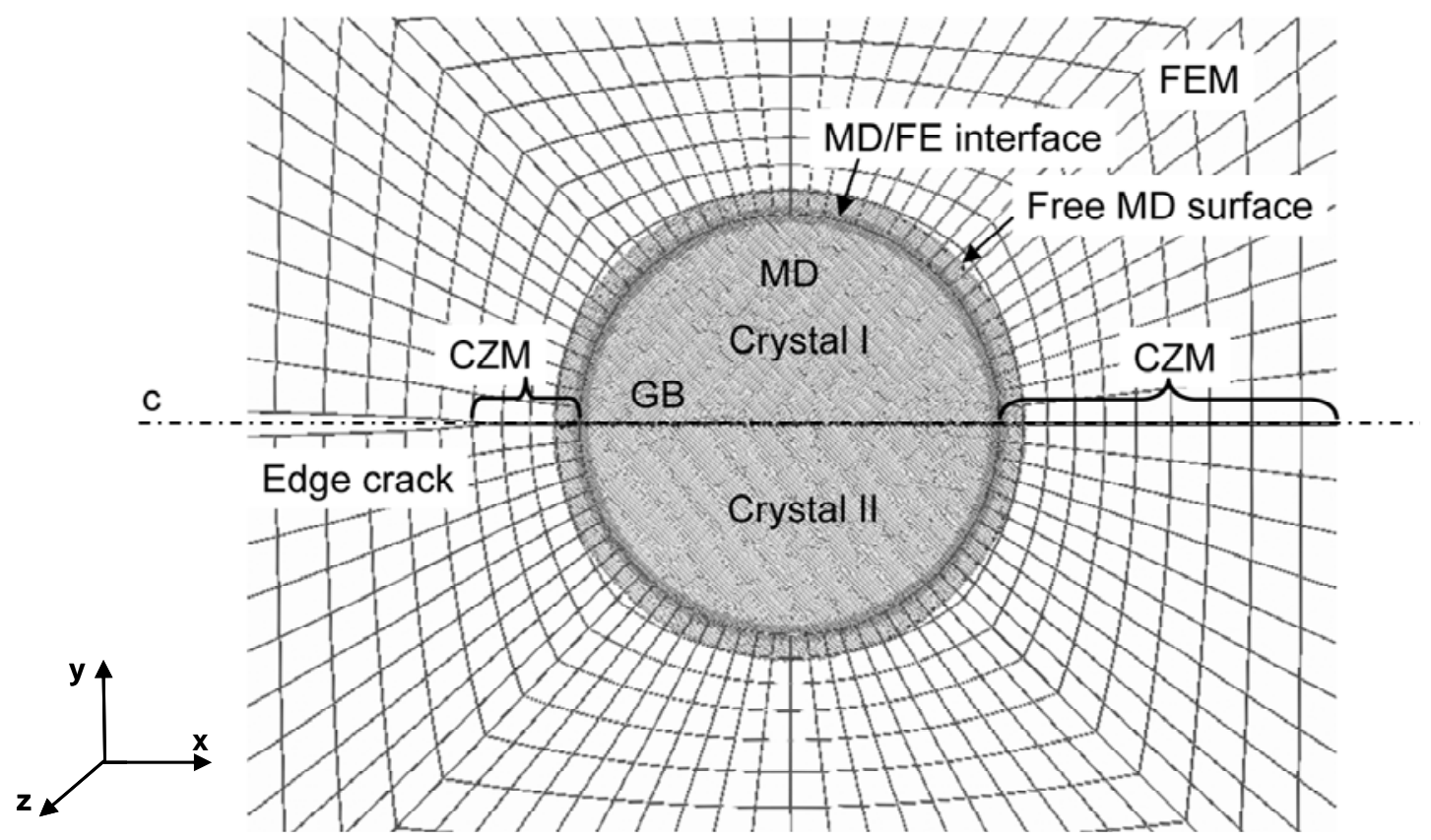

Figure 2. Configuration of the simulation model for edge-crack propagation along a grain-boundary in aluminum. 


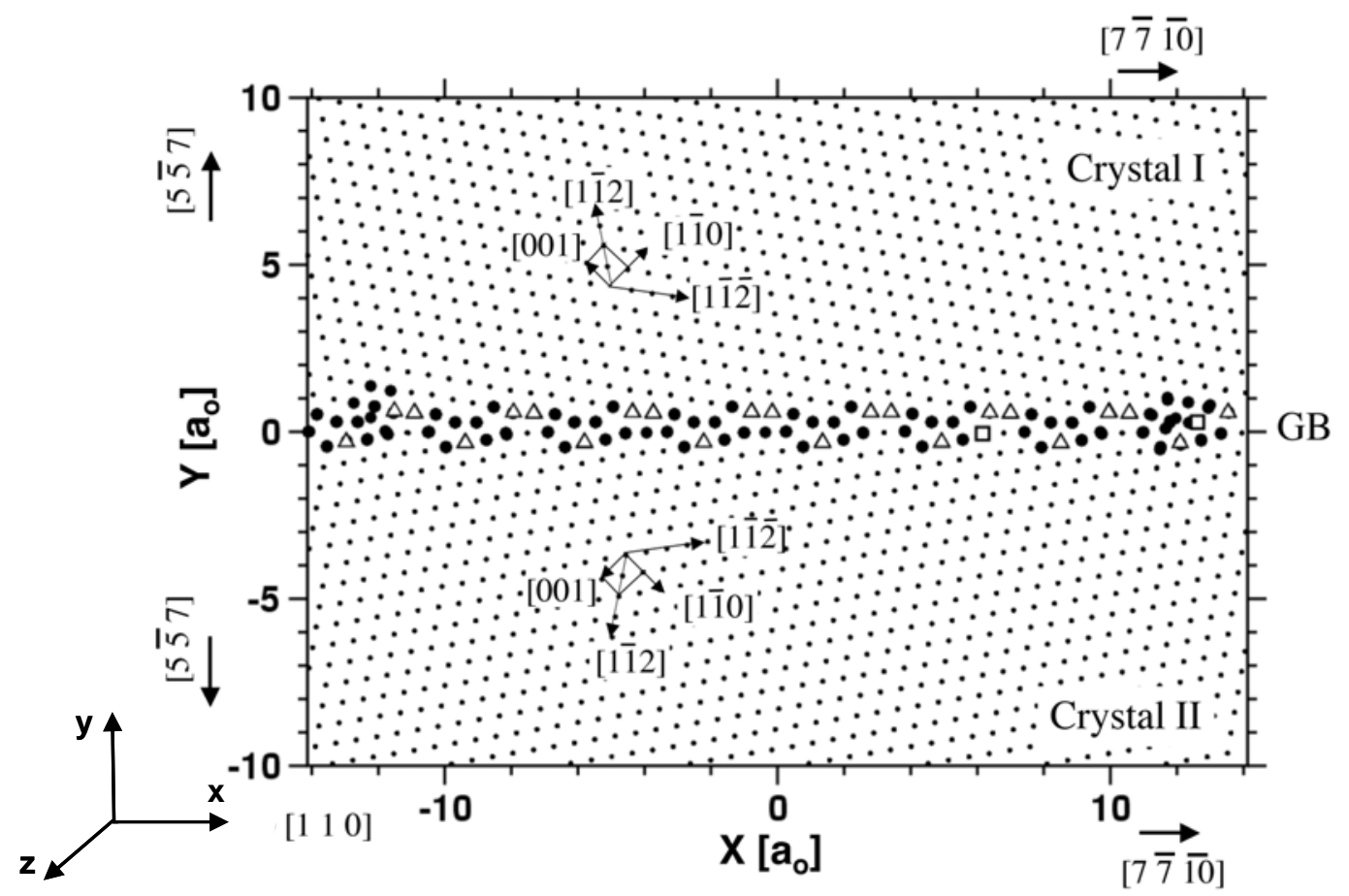

Figure 3. Crystallography and structure of the GB interface. 




Figure 4. Structure of the coupled MD-FEM ESCM approach showing the forces along the interface. 




Figure 5. Schematic diagram of the system volume slicing in the MD simulation of a $\Sigma 99$ grain boundary and defining the cohesive zone volume elements (CZVE) for extracting cohesive-zone model (CZM) parameters. 


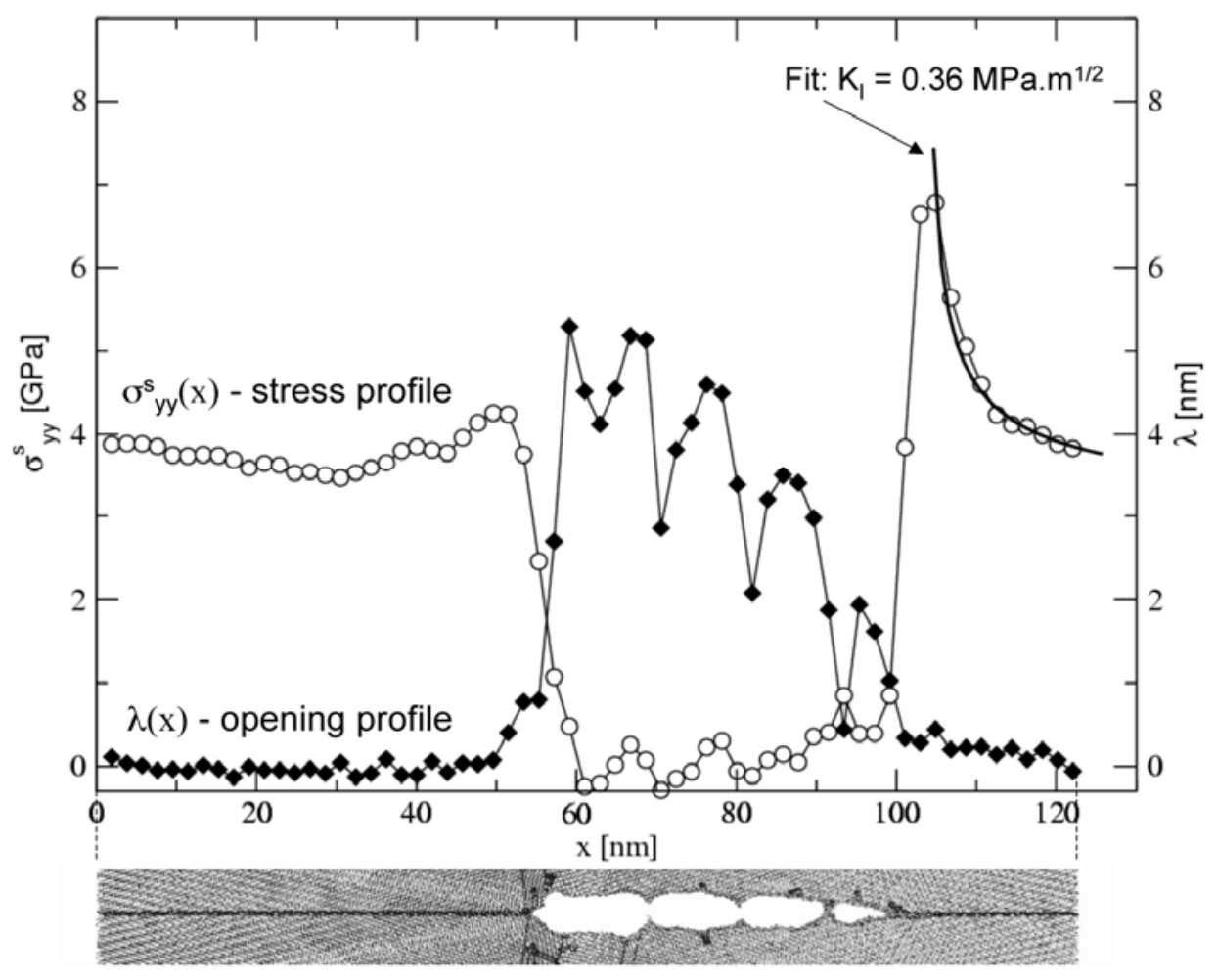

Figure 6. Stress and opening profiles extracted along the embedded crack growing in a purely MD simulation. A snapshot of the corresponding crack configuration is shown at the bottom. 


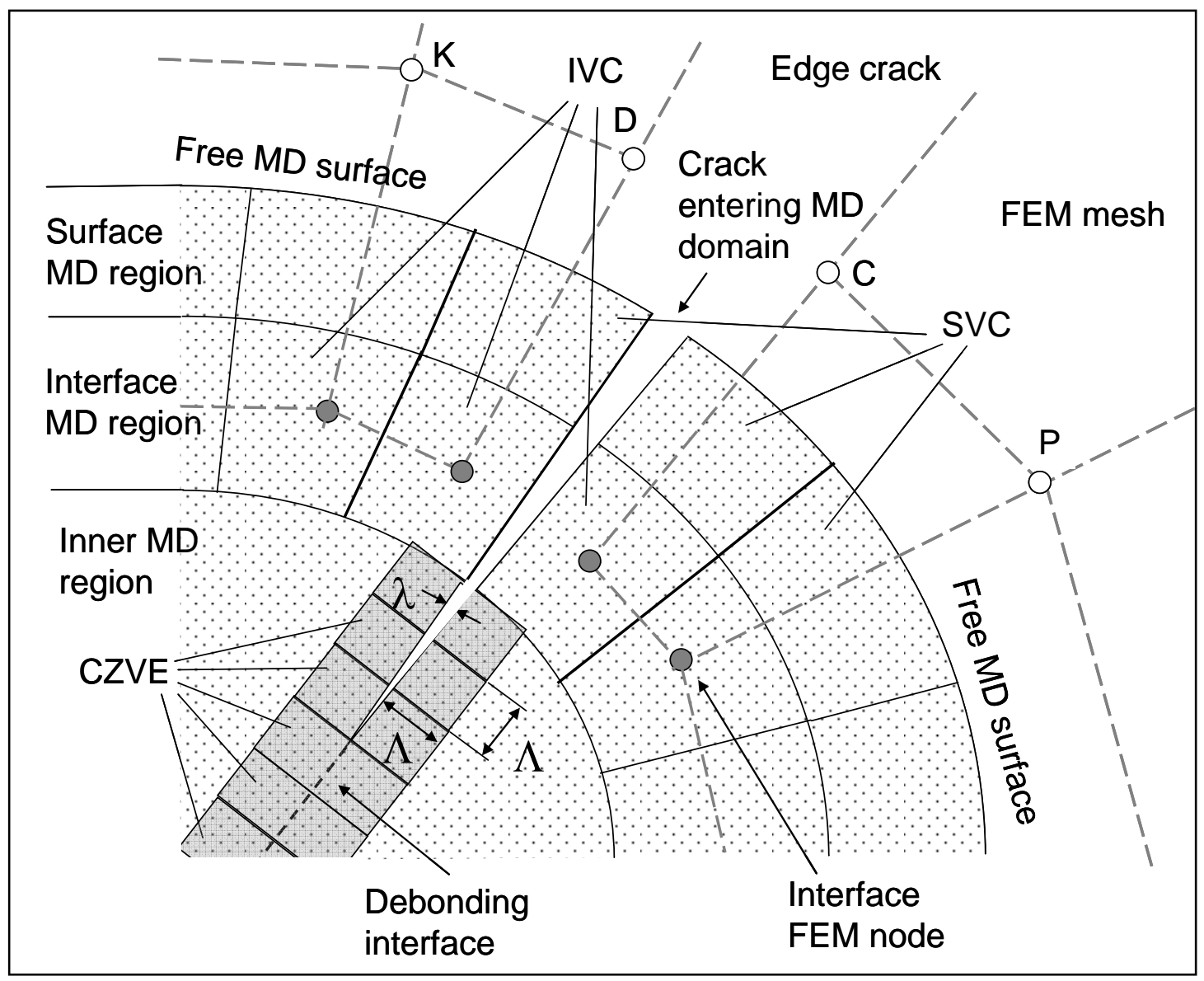

Figure 7. CZVE elements along the crack path inside the inner MD region within the ESCM. 


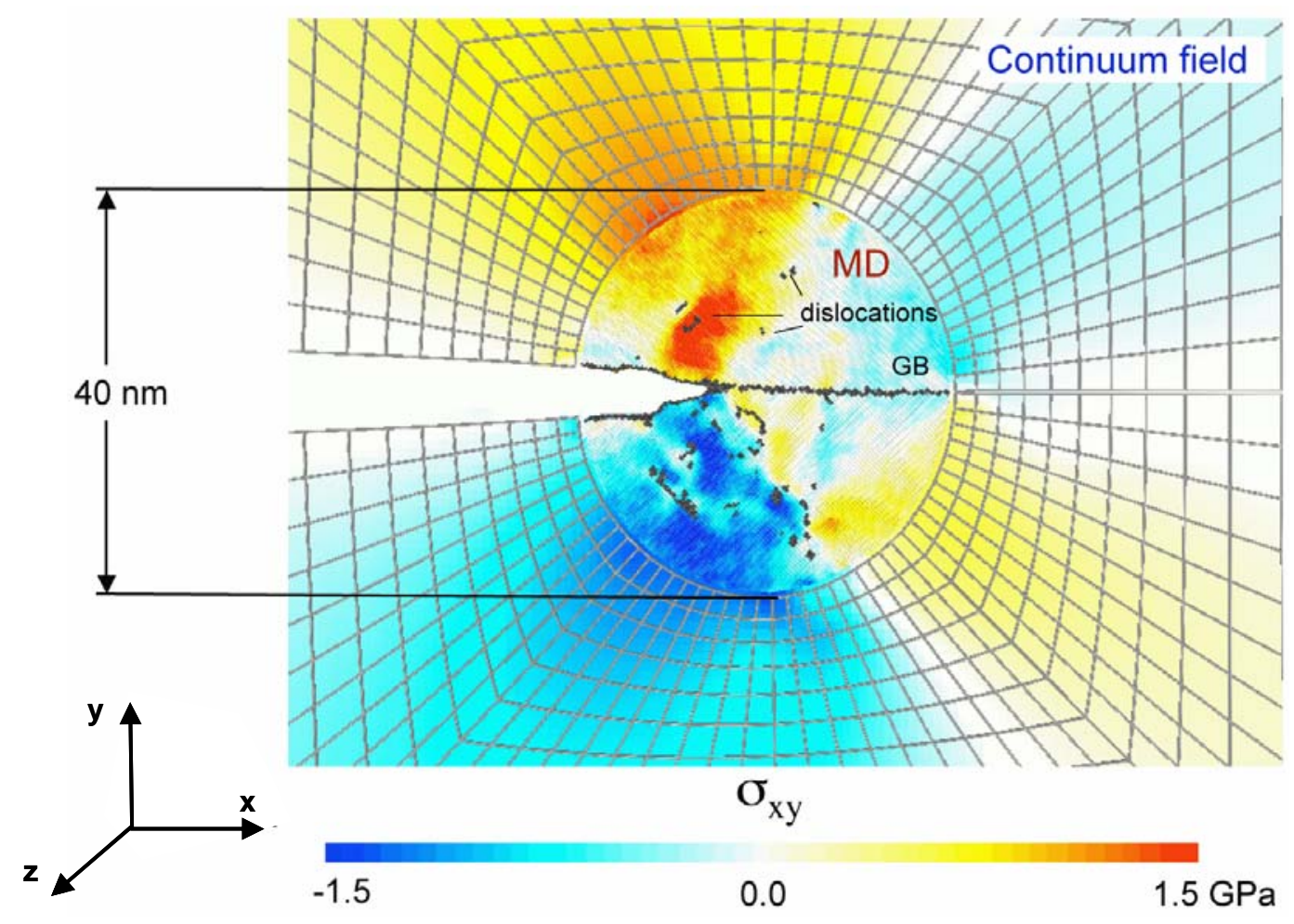

Figure 8. ESCM simulation of an edge crack propagating along a $<110>/ \Sigma 99$ symmetric tilt grain boundary in aluminum for $2 \%$ far-field uniaxial strain. 



○ fcc; O MD interface fcc; $\bullet$ hcp; $\bullet$ disordered; $\bigcirc$ surface

Figure 9. Snapshots of the ESCM model configurations simulating an edge crack propagating along a GB in a bicrystal of aluminum with CZM elements along the GB interface. 


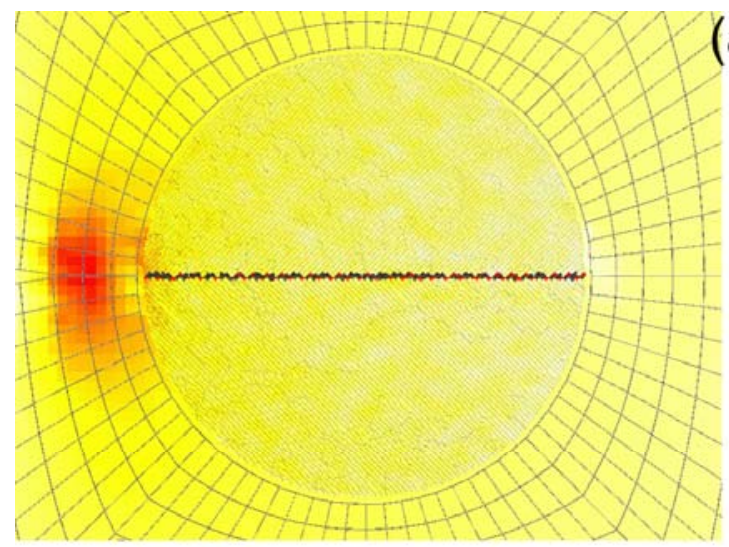

(a)
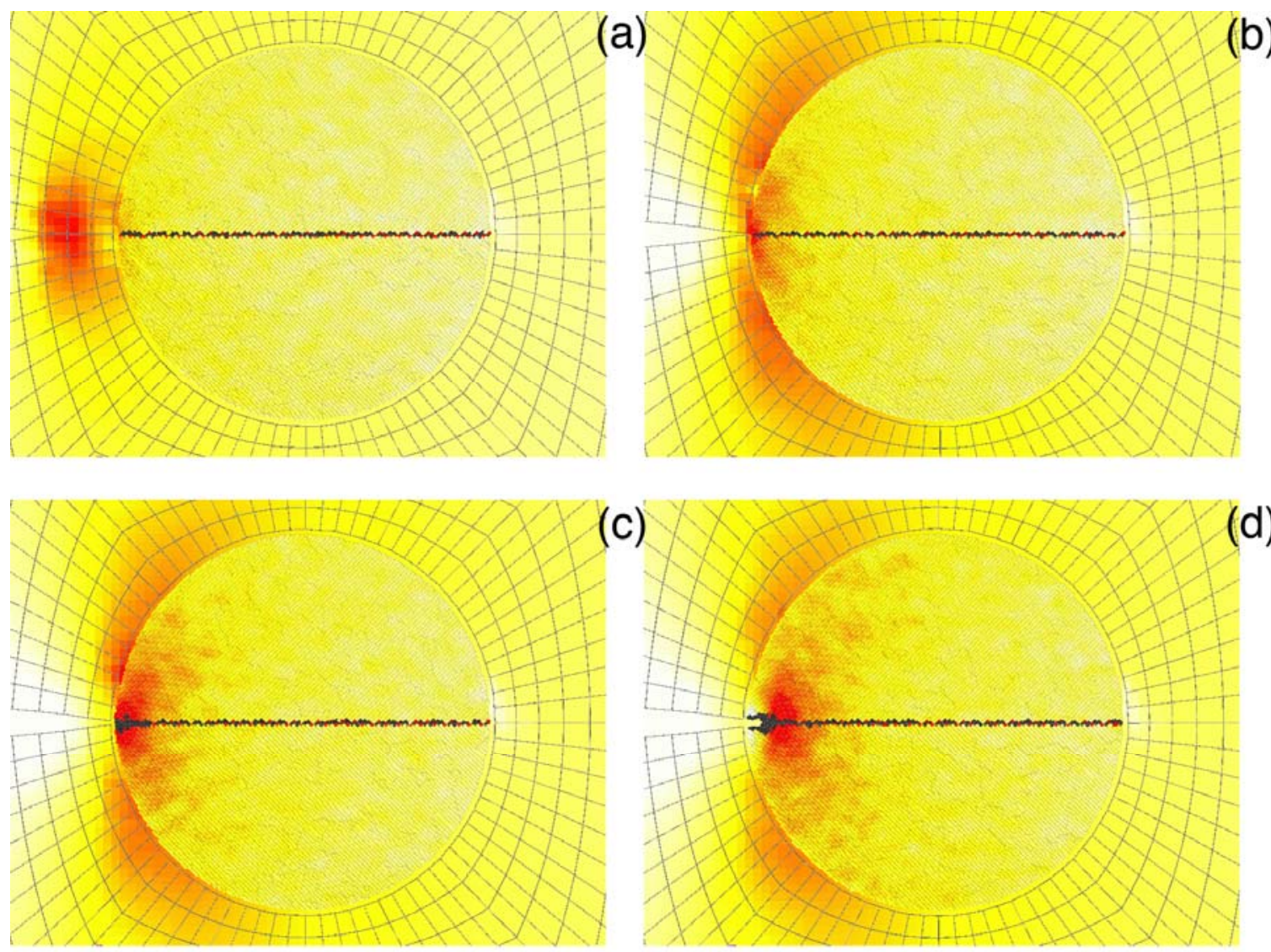

(c)

(d)
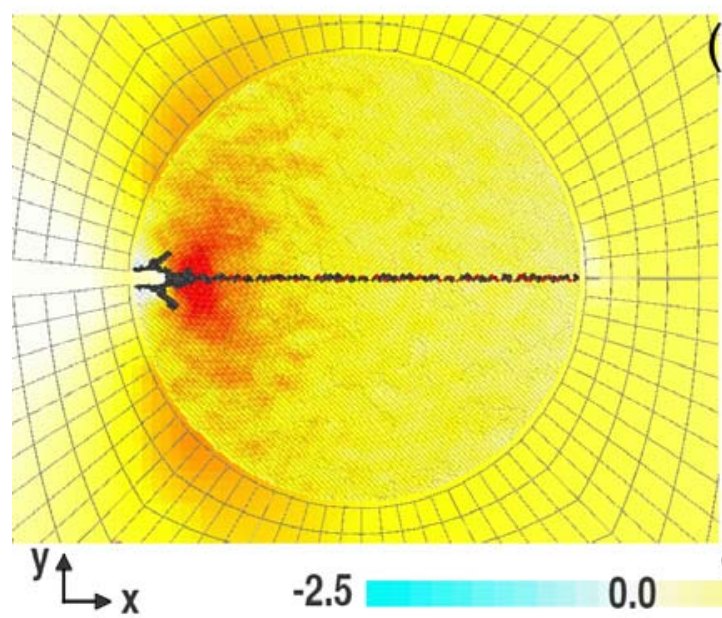

(e)
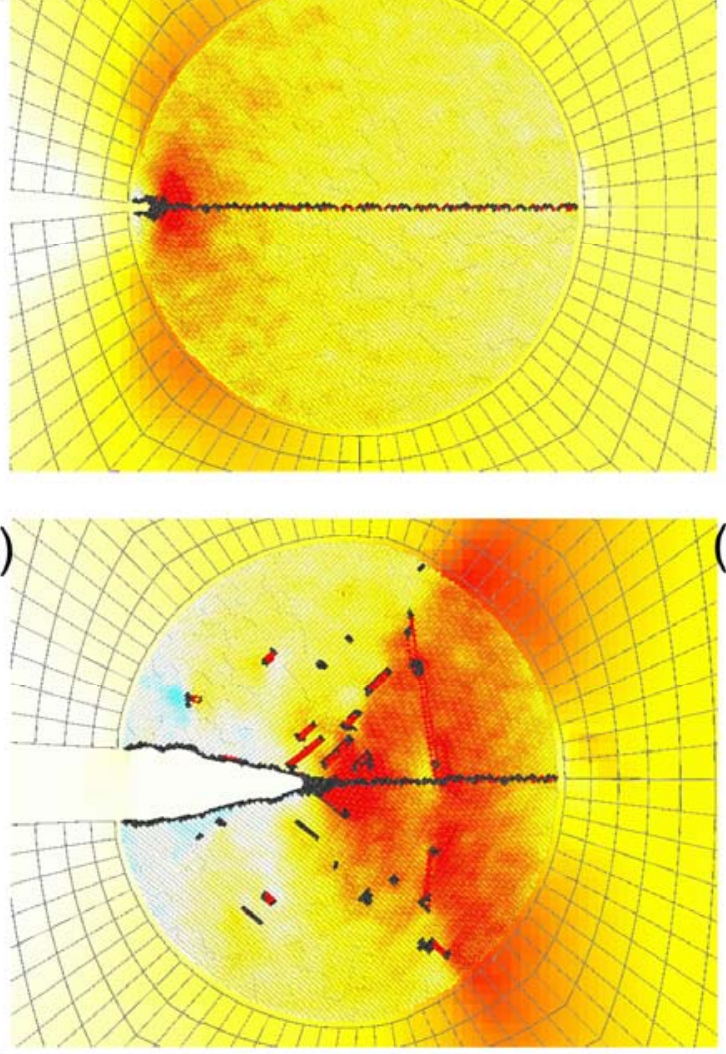

(f)

Figure 10. Stress map of $\sigma_{\text {yy }}$ corresponding to the snapshots given in Figure 9. 


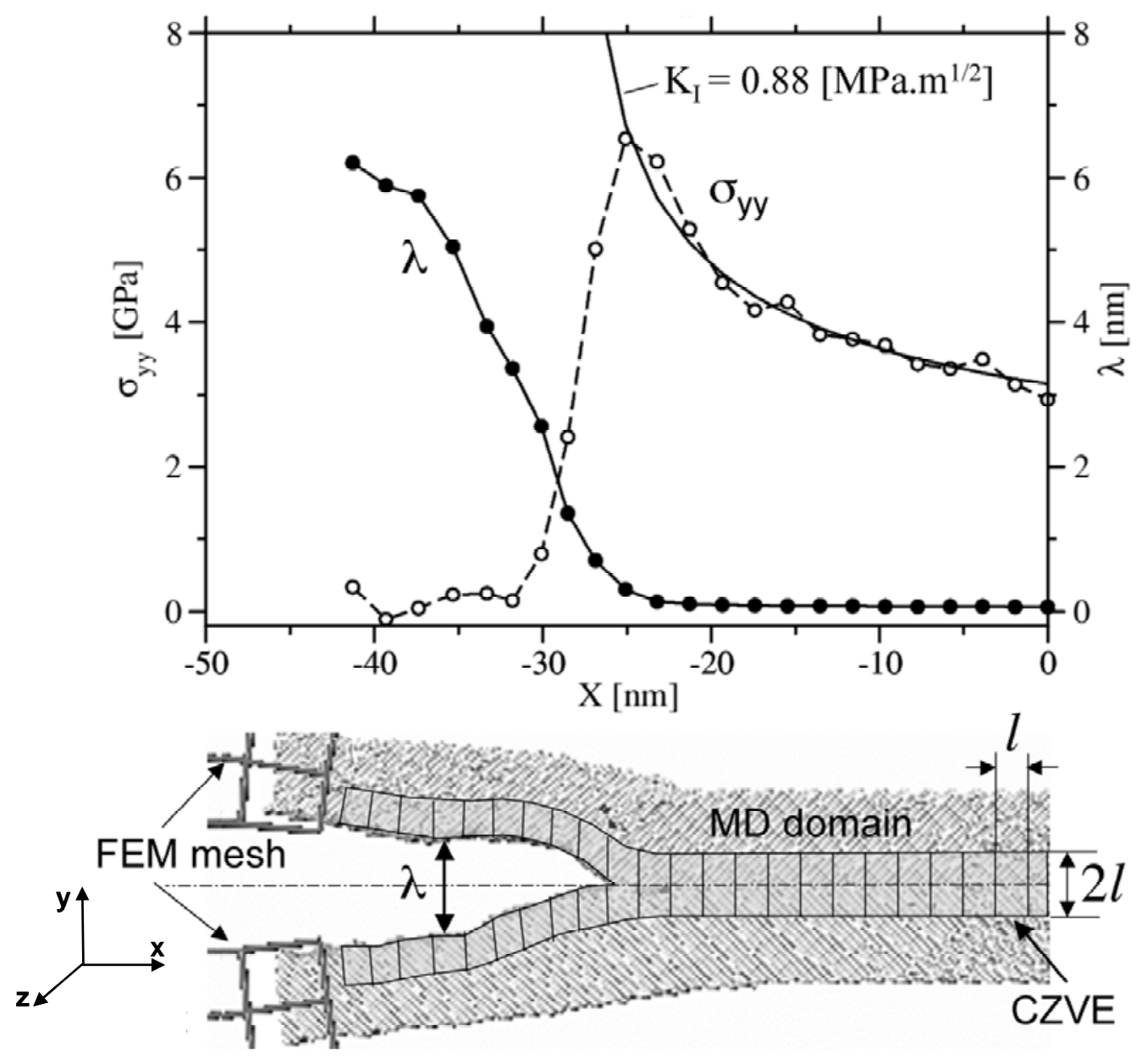

Figure 11. Stress and opening profiles extracted from cohesive-zone-volume-elements (CZVE) placed along the path of an edge crack growing along a grain-boundary inside the molecular dynamics region. 


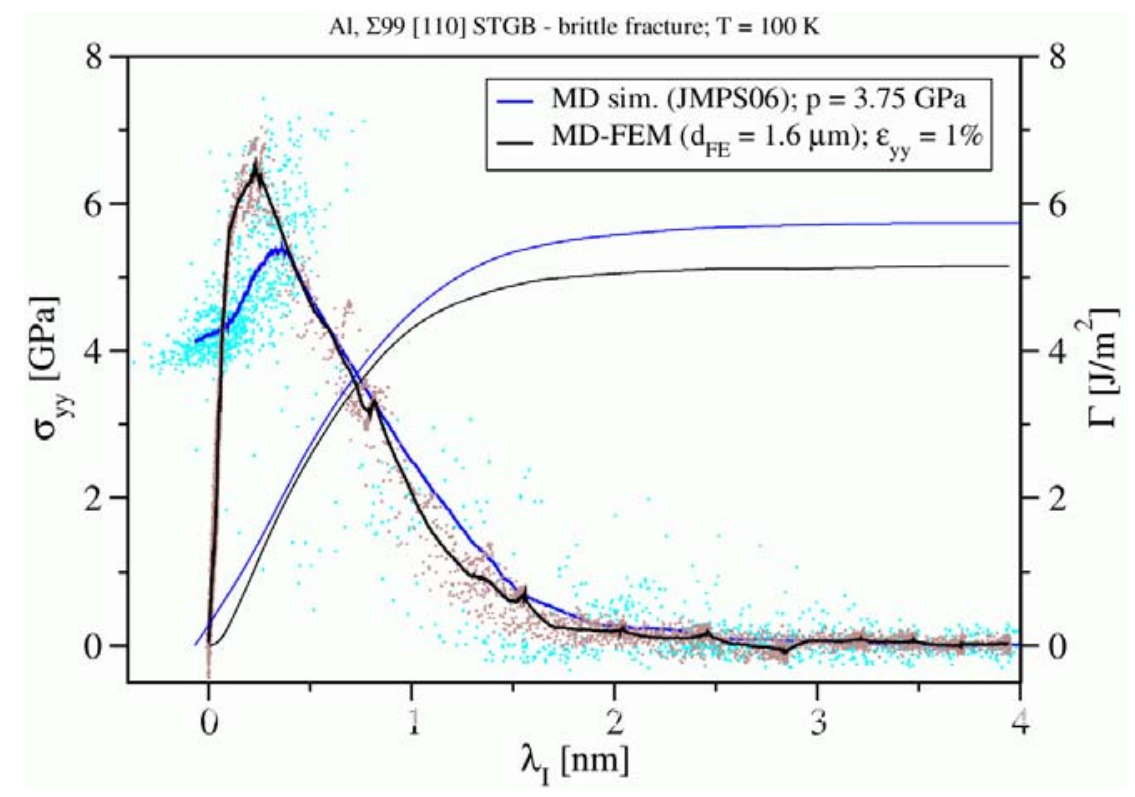

Figure 12. Comparison between the brittle CZM derived using pure MD and ESCM models. 